\title{
PAMAM-dendrimer Enhanced Antibacterial Effect of Vancomycin Hydrochloride Against Gram-Negative Bacteria
}

\author{
Azadeh Serri ${ }^{1}$, Arash Mahboubi ${ }^{2}$, Afshin Zarghi $^{3}$ and Hamid R. Moghimi ${ }^{1}$ \\ 1. Department of Pharmaceutics and Nanotechnology, School of Pharmacy, Shahid Beheshti University of Medical Sciences, \\ Tehran, Iran; 2. Food Safety Research Center, Shahid Beheshti University of Medical Sciences, Tehran. Iran; \\ 3. Department of Pharmaceutical Chemistry, School of Pharmacy, Shahid Beheshti University of Medical Sciences, Tehran, \\ Iran.
}

Received, November 8, 2018; Revised, December 7, 2018; Accepted, December 7, 2018; Published, December 13, 2018.

\begin{abstract}
Purpose: The antibacterial activity of some antibiotics is specific to either Gram-positive or Gramnegative bacteria. There are different mechanisms behind such insensitivities like inability of antibiotics to permeate through some bacterial membranes, as is the case for vancomycin in Gram-negative bacteria. The present investigation tries to overcome this problem by dendrimers, in order to make Gram-negative bacteria responsive to vancomycin. Methods: The effects of generations $3(\mathrm{G} 3)$ and $5(\mathrm{G} 5)$ polyamidoamine amine-terminated dendrimers (NH2-PAMAM), on the antibacterial activity of vancomycin, were evaluated. Vancomycin-PAMAM dendrimers complexes were prepared and their antibacterial activities were evaluated by determination of their "minimum inhibitory concentration (MIC)", "minimum bactericidal concentration" and "fractional inhibitory concentration index" values against two Gram-positive and four Gram-negative bacteria, using broth microdilution method. The complexation of vancomycin and dendrimers was also assessed by in vitro release studies across dialysis tubing using a developed HPLC method. Results: Results showed that vancomycin solution was effective against Gram-positive bacteria, but, was not effective in Gram-negative ones. Vancomycin-PAMAM dendrimers exhibited significant antibacterial efficacy against Gram-negative bacteria resulting in a decline of vancomycin MIC values by about 2, 2, 4 and 64 times in E. coli, K. pneumonia, S. typhimurium and $P$. aeruginosa, respectively. Results also showed that enhanced effect by G5 is more than G3. Dendrimers did not affect antibacterial activity of vancomycin in Gram-positive bacteria, as no permeation problem exists here. Conclusions: The present study revealed that both G3 and G5 cationic PAMAM dendrimers are able to make Gram-negative bacteria sensitive to vancomycin, resulting in decline of MIC values up to 64 times, possibly by increasing its permeation through bacterial membrane. These results look promising for broadening the antibacterial spectrum of vancomycin and such a strategy might be used for increasing the overall life of antibiotics.
\end{abstract}

\section{INTRODUCTION}

Some antibiotics show specificity towards either Gram-positive or Gram-negative bacteria. In other words, some bacteria are insensitive (resistant) to some antibiotics. This is one of the most challenging issues that human is facing todays. In fact, according to the Centers for Disease Control and Prevention, only in the United States, 2 million people are infected with insensitive bacteria and at least 23000 people die as a result of such infections annually (1). The estimated economic burden of antibioticresistant infections on the USA economy is more than $\$ 20$ billion per year as direct health care costs, and as much as $\$ 35$ billion indirectly in lost productivity from hospitalizations and disability until recuperation (1). On the other hand, developing rate of new antibiotics is prominently declining (2).
Infectious Diseases Society of America highlighted the serious paradox in fighting antibiotic resistance and stated that "the pharmaceutical pipeline is drying up" because the pharmaceutical companies are losing interest in developing new antibiotics. The main reason of this phenomenon is the high cost and long period of the new antibiotic production process (3), which requires an average investment of at least $\$ 2.6$ billion and 10 years (4). In addition, these drugs simply are not as profitable as drugs that treat chronic (long-term) health problems.

Corresponding author: Hamid R. Moghimi, School of Pharmacy, Shahid Beheshti University of Medical Sciences, Valiasr Ave., Niayesh Junction, P.O Box: 14155-6153, Tehran, Iran; Phone: +98 21 88665317; E-mail: hrmoghimi@sbmu.ac.ir 
In such a condition, improving the antibacterial efficacy of available antibiotics by broadening their spectrum of activity and increasing their effectiveness can reduce or even solve the problem, as is investigated here for vancomycin against Gramnegative bacteria.

Vancomycin is a large glycopeptide antibiotic which is the choice in the treatment of resistant Gram-positive bacteria but is not effective in Gramnegative bacteria (5). The glycopeptide forms high affinity complexes with terminal D-Alanine-DAlanine dipeptides in peptidoglycan wall of bacteria (6), which sterically interferes with the assembly of the bacterial cell wall (7), resulting in osmotic lysis upon disturbance in cell wall completion and eventually cell death (8).

As both Gram-positive and Gram-negative bacteria have the peptidoglycan cell wall (9), theoretically vancomycin should affect both but practically, only Gram-positive bacteria are sensitive to this drug. Gram-negative bacteria are insensitive to many antibiotics including vancomycin because they have an additional outer membrane in contrast to the Gram-positive ones. Outer membrane which is the major permeability barrier in these bacteria is comprised of an asymmetric bilayer of lipopolysaccharide (LPS) and phospholipids, and has many nonspecific porins and specific uptake channels (10). These bilayers are more rigid than normal bilayers, slowing passive diffusion of hydrophobic compounds, on the other hand, porin channels are available for hydrophilic drugs, but their narrow pores limit the penetration of drugs by size such that only hydrophilic drugs with a molecular weight of less than 600 Da can pass through them and enter to the bacteria (11). Vancomycin which is a large hydrophilic glycopeptide, with a molecular weight around 1400 $\mathrm{Da}$, cannot diffuse through these porin channels. Therefore, does not show required antimicrobial activity in these bacteria (12), due to this permeation barrier, while Gram-positive bacteria lack this outer membrane layer and thus, the drug reach its site of action (peptidoglycan wall) directly and are effective against Gram-positive bacteria like S. aureus and methicillin-resistant $S$. aureus.

One of the methods used for overcoming permeation barriers is optimization of drug delivery systems including application of nanoparticles. Different nanoparticles are used in drug delivery including liposomes, metallic nanoparticles, polymeric nanoparticles and carbon nanotubes and have been suggested for application in antimicrobial drug delivery (13, 14), among which polyamidoamine dendrimers (PAMAM) were used in the present investigation.

Dendrimers are highly branched, globular molecules, usually referred to as "unilamellar micelles" with a very narrow molecular distribution and nano-scale size. Dendrimers provide a three dimensional architecture with void cavities in the internal part and many functional branches grow radially from the core. These properties make it possible to either encapsulate a drug within interior cavity or to the highly symmetric branched outer layer through either covalent bonding or noncovalent complexation (15-17). Among all dendrimers, PAMAM dendrimers are widely used as drug or gene carriers (18). PAMAM dendrimers can permeate across epithelial barriers of the gastrointestinal tract and as a result are potential drug carriers for oral drug delivery (19), as well as nonviral gene delivery vectors because they can condense DNA and protect it from in vivo degradation (20). PAMAM dendrimers were also utilized in other fields of drug delivery such as photodynamic therapy (21), and biosensors (22). PAMAM dendrimers show toxicity as a function of surface charge, concentration, exposure-time, functional groups and generation $(19,23)$. A dose escalation study determined maximum tolerated doses of dendrimers and revealed that the dendrimers were safe for oral administration except for NH2and $\mathrm{OH}$-terminated generation 7 PAMAM, which exhibited signs of toxicity at relatively low dosage (24). Higher generation PAMAM dendrimers are more toxic than the lower ones (25). As a result, there is a safe non-toxic margin to use them as oral drug carriers, furthermore, surface modification like shielding with PEG (26), or shielding by liposomes, which was done by our group $(27,28)$, can be used to overcome toxicity limitation of dendrimers in vivo. In this investigation generations 3 and 5 (G3 and G5) PAMAM dendrimers are used.

Many studies have used dendrimers, merely based on two strategies: 1) dendrimers as permeability enhancers and 2) dendrimers for solubility enhancement of poorly soluble drugs. Cheng et al. (29) used generation 4 PAMAM dendrimers as drug carrier for quinolones, (nadifloxacin and plurifloxacin) and showed the capability of this dendrimer to increase antibacterial activities of plurifloxacin against E. coli, and promote their solubility in aqueous solution. Ma and 
co-workers (30), also revealed the same results for sulfamethoxazole against $E$. coli. Winnika et al. (31), using G3 and G5 dendrimers to enhance water solubility of erythromycin and tobramycin.

In the present study we investigated the possibility of broadening the spectrum of activity of vancomycin to Gram-negative bacteria. To the best of our knowledge, there is not such an investigation reported in the literature. However, there are two covalently bound vancomycin-dendrimer systems studied on Gram-positive bacteria $(32,33)$, in order to change its interaction with di-peptide bridge in cell wall's peptidoglycan for formation prevention, completely different from our hypothesis.

\section{MATERIALS}

\section{Chemicals and media}

Amine-terminated third and fifth-generation polyamidoamine (PAMAM) dendrimers (G3-NH2 and G5-NH2), having ethylenediamine cores were obtained as $20 \%$ and $5 \%$ methanolic solutions (Sigma-Aldrich Chemie GmbH, Germany). Vancomycin hydrochloride (called vancomycin here), was purchased from Gold Biotechnology INC. (USA). Polyethersulfone (PES) membrane filters were acquired from JET Bio-Filtration Co., Ltd (China). Muller-Hinton agar (MHA) and MullerHinton broth (MHB) were purchased from Merck (Germany).

\section{Microorganisms:}

Gram-positive bacteria including Staphylococcus aureus ATCC (the American Type Culture Collection) 6538, Methicillin resistant Staphylococcus aureus (MRSA) ATCC 33591 and Gram-negative bacteria including Escherichia coli ATCC 8739, Klebsiella pneumonia ATCC 10031, Salmonella typhimurium ATCC 14028 and Pseudomonas aeruginosa ATCC 9027 were obtained from Iranian Research Organization for Science and Technology, Persian Type Culture Collection (PTCC), Tehran, Iran.

\section{Development and validation of a stability indicating HPLC method for vancomycin hydrochloride}

A stability indicating HPLC method was developed and validated to determine vancomycin and its degradation products in different situation such as acidic, oxidative and basic conditions, heat and sunlight exposure. The separation was achieved using a Capital C8-Optimal column $(250 \times 4.6 \mathrm{~mm}$, i.d., $5 \mu \mathrm{m}$ particle size) with a mobile phase composed of citrate buffer ( $\mathrm{pH} 4$ ), acetonitrile and methanol in the ratio of $85: 10: 5$ (by volume), respectively (34). Cephalexin was used as internal standard (IS).

\section{Preparation of PAMAM dendrimer solution}

Methanol was evaporated from the supplied methanolic dendrimer solution under a stream of dry nitrogen gas at room temperature. The obtained solid dendrimer was then re-suspended in deionized water to obtain an aqueous stock solution of $10 \mathrm{mg} / \mathrm{mL}$ (35).

Preparation of vancomycin-PAMAM dendrimers Dried dendrimer was re-suspended in vancomycin hydrochloride aqueous solution, containing 10 $\mathrm{mg} / \mathrm{mL}$ vancomycin (based on its inhibitory concentration) and $10 \mathrm{mg} / \mathrm{mL}$ of either G3 or G5. Vancomycin-PAMAM dendrimer mixture was then stirred for up to 1 hour for drug-dendrimer complexation.

Evaluation of in vitro release profile of vancomycin-dendrimer

In vitro release test was carried out with dialysis membrane, in order to evaluate release profile of vancomycin PAMAM dendrimer. Vancomycin was dissolved in water at the same concentration and used as control. $1 \mathrm{~mL}$ of these solutions were transferred to a dialysis tubing (MW cut-off 3500 $\mathrm{Da}$ ) and then the dialysis tubing was placed in a 50 $\mathrm{mL}$ beaker containing $20 \mathrm{~mL}$ distilled water (30) at $37^{\circ} \mathrm{C}$. After defined intervals, $50 \mu \mathrm{L}$ of sample was withdrawn from the beaker and the receptor phase was replaced with $50 \mu \mathrm{L}$ distilled water.

The amount of drug in the receptor phase was evaluated with a HPLC method previously developed by the authors (34) and as described above. The mobile phase was pumped using an isocratic HPLC system at a flow rate of $1 \mathrm{~mL} / \mathrm{min}$ and quantification of analyte was based on measuring its peak areas at $280 \mathrm{~nm}$.

\section{Determination of minimum inhibitory concentration (MIC) and minimum bactericidal concentration (MBC) \\ MIC and MBC determination of vancomycin}

Bacterial inocula were prepared from an 18-24 h incubation of the test organism grown on MHA plate. Some colonies of each bacterial strain (total six 
microorganisms) were sampled with a swab, then transferred to MHB to get a $0.5 \mathrm{Mc}$ Farland bacterial suspension. Appropriate transmittance was adapted by reading samples in UV-vis. spectrophotometry (mini-Shimatzu, Japan) instrument at $625 \mathrm{~nm}$. The inoculum was diluted by MHB solution to reach an inoculum suspension of about $5 \times 10^{6}$ colony forming unit $(\mathrm{CFU}) / \mathrm{mL}$. Finally, $10 \mu \mathrm{L}$ of this suspension was added to each microplate well to get a final bacterial concentration of about $5 \times 10^{5} \mathrm{CFU} / \mathrm{mL}$ in each well (36).

Minimum inhibitory concentrations of vancomycin against six bacteria (two Grampositives and four Gram-negatives), were determined using broth microdilution method in accordance with clinical and laboratory standards institute guidelines $(37,38)$. Aqueous solution of vancomycin hydrochloride was prepared and transferred to each microplate well in order to obtain a two-fold serial dilution of the original stock solution (from $1: 2$ to $1: 2084$ ). After $18-24 \mathrm{~h}$ of incubation at $37^{\circ} \mathrm{C}$, the MIC was determined. Determination of the MBC was done by sub culturing $10 \mu \mathrm{L}$ of each well without any visible growth. The culturing was performed on MHA medium and incubated at $37^{\circ} \mathrm{C}$ for $24 \mathrm{~h}$. MBC was defined as the lowest concentration resulting in a negative subculture or giving no colony after incubation. The experiments were carried out in 3 replicates $(n=3)(39,40)$.

\section{MIC and MBC determination of PAMAM dendrimers}

To conduct the antibacterial activity studies, $100 \mu \mathrm{L}$ MHB medium was first distributed from the first to the twelfth well of a 96-well plate. Then, $100 \mu \mathrm{L}$ of test solutions (PAMAM dendrimers), prepared as mentioned above, were added to the first test well, and subsequently, $100 \mu \mathrm{L}$ of scalar dilution was transferred from the second to the eleventh well, the twelfth well was added without any drug. Finally, 10 $\mu \mathrm{L}$ of a microbial suspension was added to each well of the plate. All the formulations were filtered through a 0.22-micron PES membrane filters in order to make them free of any possible contamination prior to each microbial test. The plates were incubated for $18-24 \mathrm{~h}$ at $37^{\circ} \mathrm{C}$. The MICs were assessed where there was no visible growth of bacteria. MBC studies were performed as described in the previous section for vancomycin hydrochloride.
MIC and MBC determination of vancomycin PAMAM dendrimers

The MICs of vancomycin formulations (vancomycin-PAMAM dendrimers containing 10 $\mathrm{mg} / \mathrm{mL}$ PAMAM dendrimers and $10 \mathrm{mg} / \mathrm{mL}$ vancomycin) were assessed with the same abovementioned procedure. Aqueous solution of vancomycin dendrimers was transferred to each microplate well in order to obtain a two-fold serial dilution of the original stock solution (from 1:2 to 1:2084). The MICs were determined where there was no visible growth of bacteria. MBC studies were performed as described in the previous section for vancomycin hydrochloride.

Subsequently the fractional inhibitory concentrations (FIC) and fractional inhibitory concentrations index (FICI) were calculated in order to identify vancomycin-dendrimer interaction mechanisms, as follows:

$\mathrm{FIC}_{\mathrm{A}}=(\mathrm{MIC}$ of agent $\mathrm{A}$ in combination $) /(\mathrm{MIC}$ of agent $\mathrm{A}$ alone)

$\mathrm{FIC}_{\mathrm{B}}=(\mathrm{MIC}$ of agent $\mathrm{B}$ in combination $) /(\mathrm{MIC}$ of agent $\mathrm{B}$ alone)

$\sum \mathrm{FIC}=\mathrm{FICI}=\mathrm{FIC}_{\mathrm{A}}+\mathrm{FIC}_{\mathrm{B}}$

\section{Stability studies}

As it was mentioned previously, a stability indicating HPLC method was developed and validated by authors, to determine vancomycin stability in different harsh conditions and to identify its degradative products. Further experiments were carried out in order to evaluate the stability of this complex, as described below.

\section{Determination of vancomycin aqueous solution stability}

Vancomycin UV-spectra was obtained when it was dissolved in deionized water and in PBS (pH: 7.4). Subsequently, its HPLC determination was carried out in both media to determine any difference in its chromatogram. Furthermore, the amount of vancomycin hydrochloride in both aqueous and physiological media was evaluated after leaving the solution at room temperature for one month.

\section{Determination of Vancomycin PAMAM dendrimer stability}

In order to evaluate vancomycin PAMAM dendrimer stability, the preparation was kept at room 
temperature for 1 month and then was assayed by HPLC to determine any change in concentration. Data were then analyzed statistically using one-way ANOVA test, considering $\mathrm{P}<0.05$ as significant, followed by post hoc Tukey's HSD test to determine the differences between means.

\section{RESULTS}

\section{In vitro release studies of vancomycin-PAMAM dendrimers}

Figure 1 shows $24 \mathrm{~h}$ release profiles of vancomycin from G3 and G5 PAMAM dendrimers and vancomycin solution. As it is shown, after $24 \mathrm{~h}, \mathrm{G} 3$ and G5 PAMAM-containing systems, released about $63 \%$ and $44 \%$ of vancomycin, respectively, while vancomycin was totally released from solution in 1 $h$. These data reveal a sustained release manner for vancomycin-dendrimer systems in comparison to vancomycin solution and that, G5 dendrimer released less vancomycin during $24 \mathrm{~h}$ release than G3 system.

\section{MIC and MBC of vancomycin solution}

Table 1 shows MIC and MBC values for vancomycin hydrochloride obtained by broth microdilution method. Staphylococcus aureus is considered vancomycin-resistant at MIC values of higher than $16 \mu \mathrm{g} / \mathrm{mL}(37,38)$. Based on this, vancomycin hydrochloride was effective only on Gram-positive bacteria. The most sensitive microorganism, with minimum MIC value, was $S$. aureus with an MIC of $2.4 \mu \mathrm{g} / \mathrm{mL}$ while the highest values were for $P$. aeruginosa with an MIC and MBC value of 5000 and $40000 \mu \mathrm{g} / \mathrm{mL}$, respectively. The MIC value for Gram-negative bacteria was more than $156.2 \mu \mathrm{g} / \mathrm{mL}$.

\section{MIC determination of PAMAM dendrimers}

Table 2 shows G3 and G5 PAMAM dendrimer MIC values. PAMAM dendrimers (without incorporated drug), in both generation, were inactive against both Gram-positive and Gram-negative bacteria. The MIC values for Gram-positive bacteria in both generations were less than MIC values of Gramnegative bacteria. The minimum MIC was for G3 dendrimers and on $S$. aureus $(1250 \mu \mathrm{g} / \mathrm{mL})$. The $\mathrm{MBCs}$ were very high in both generations (more than $20000 \mu \mathrm{g} / \mathrm{mL}$ ).

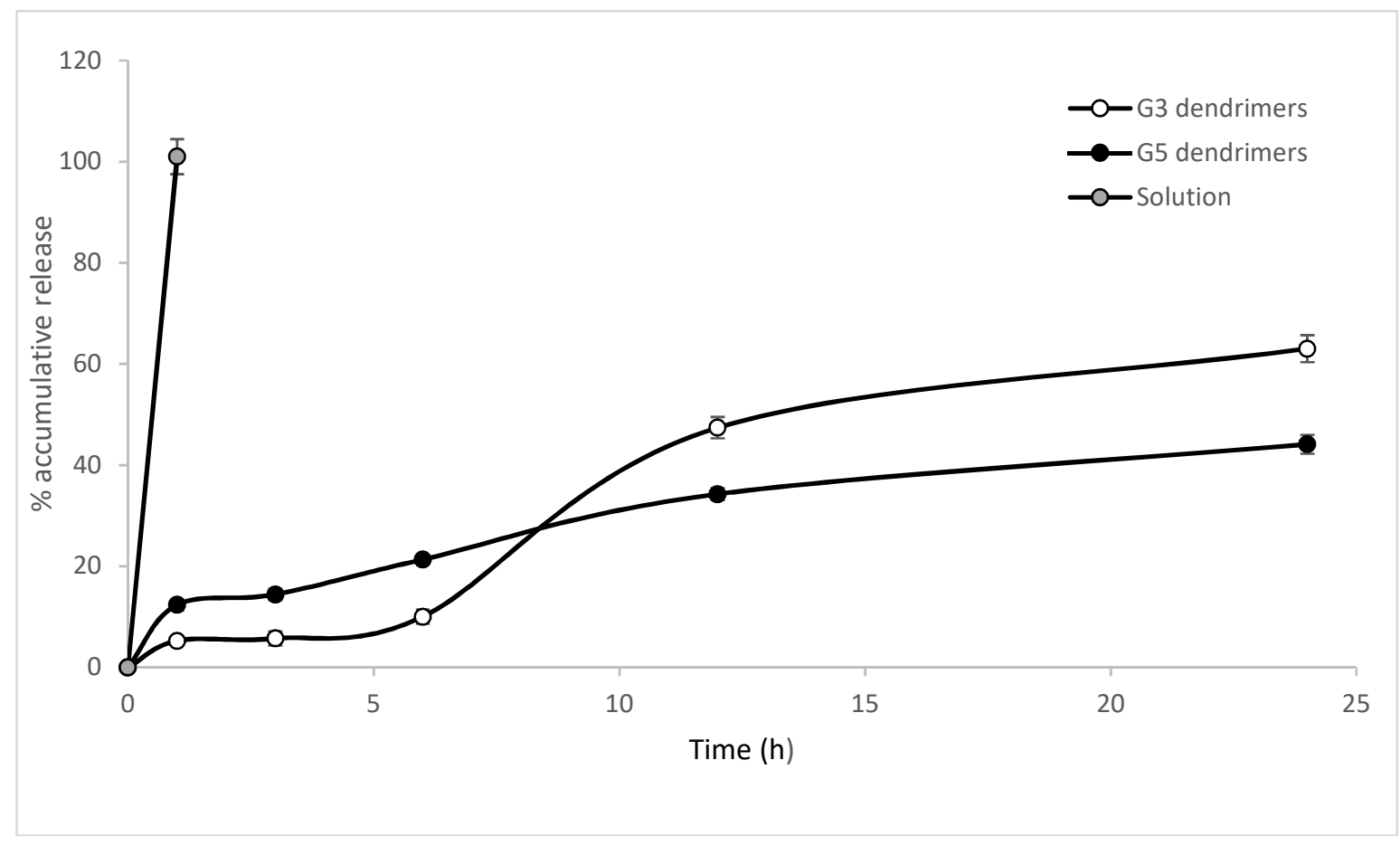

Figure 1. Cumulative release of vancomycin from G3 and G5 dendrimer systems and solution over $24 \mathrm{~h}$. Data are mean \pm $\mathrm{SD}(\mathrm{n}=3)$. (The error bars are within the size of the symbols where not visible). 
Table 1. Minimum Inhibitory Concentration (MIC) and Minimum Bactericidal Concentration (MBC) $(\mu \mathrm{g} / \mathrm{mL})$ values of vancomycin hydrochloride solution $(\mathrm{n}=3)^{*}$

\begin{tabular}{llll}
\hline Microorganism & ATCC & MIC* & MBC* \\
\hline S. aureus & 6538 & 2.4 & 2.4 \\
MRSA & 33591 & 4.8 & 9.6 \\
E. coli & 8739 & 156.2 & 156.2 \\
K. pneumonia & 10031 & 625 & 625 \\
S. typhimurium & 14028 & 625 & 625 \\
$P$. aeruginosa & 9027 & 5000 & 40000 \\
\hline
\end{tabular}

*: All tests were run in triplicates and same MIC (or MBC) values were observed in the replications.

Table 2. MIC and MBC values of G3 and G5 PAMAM dendrimer $(\mu \mathrm{g} / \mathrm{mL})(\mathrm{n}=3)^{*}$

\begin{tabular}{llccc}
\hline Bacteria & \multicolumn{2}{c}{ G3 } & MIC & G5 \\
\cline { 2 - 5 } S. aureus & MIC & MBC & 2500 & MBC \\
MRSA & 1250 & $>20000$ & 2500 & $>2000$ \\
E. coli & 2500 & $>20000$ & 20000 & $>20000$ \\
K. pneumonia & 5000 & $>20000$ & 20000 & $>20000$ \\
S. typhimurium & 20000 & $>20000$ & 20000 & $>20000$ \\
$P$. aeruginosa & 20000 & $>20000$ & 20000 & $>20000$ \\
\hline
\end{tabular}

*: All tests were run in triplicates and same MIC (or MBC) values were observed in the replications.

\section{MIC determination of vancomycin-dendrimer complexes}

Table 3 shows G3 and G5 vancomycin-PAMAM dendrimer complexes MIC values. VancomycinPAMAM dendrimers, in both cases (G3 and G5), significantly potentiated the drug effect on almost all Gram-negative bacteria. It reduced vancomycin MIC from 156.2 to $78.1 \mu \mathrm{g} / \mathrm{mL}$, from 625 to $156.2 \mu \mathrm{g} / \mathrm{mL}$ and from 5000 to $78.1 \mu \mathrm{g} / \mathrm{mL}$ for E. coli, $S$. typhimurium and $P$. aeruginosa, respectively. $P$. aeruginosa with an MIC of $78.1 \mu \mathrm{g} / \mathrm{mL}$ was the most sensitive bacteria to the both dendrimer formulations. No MIC change was observed for the effects of vancomycin-containing dendrimers on Gram-positive bacteria, compared to vancomycin solution. On the other hand, dendrimers had much higher MIC values for both Gram-positive and Gram-negative bacteria, in comparison to vancomycin-PAMAM dendrimers.

Table 4 provides FIC values for vancomycindendrimer systems, interpreted as synergism $(\mathrm{FICI} \leq$ $0.5)$, partial synergism $(0.5<$ FICI $<1)$ and indifference or additive $(1 \leq \mathrm{FICI} \leq 4)$, based on criteria described by (41). For both G3 and G5 vancomycin dendrimers, the minimum FICI was related to $P$. aeruginosa, with an FICI of about 0.02 which means there is a strong synergism $(\mathrm{FICI} \leq 0.5)$ between vancomycin and dendrimers on this bacterium.

Table 3. Vancomycin-PAMAM dendrimers MIC and MBC values $(\mu \mathrm{g} / \mathrm{mL})(\mathrm{n}=3)^{*}$

\begin{tabular}{|c|c|c|c|c|c|c|}
\hline \multirow[t]{2}{*}{ Bacteria } & \multicolumn{2}{|c|}{$\begin{array}{c}\text { Vancomycin } \\
\text { (Control) }\end{array}$} & \multicolumn{2}{|c|}{ Vancomycin/G3 ${ }^{a}$} & \multicolumn{2}{|c|}{ Vancomycin/G5 ${ }^{\mathrm{b}}$} \\
\hline & MIC & MBC & MIC & MBC & MIC & MBC \\
\hline S. aureus & 2.4 & 2.4 & 2.4 & 4.8 & 2.4 & 19.2 \\
\hline MRSA & 4.8 & 9.6 & 4.8 & 9.6 & 4.8 & 156.2 \\
\hline E.coli & 156.2 & 156.2 & 78.1 & 78.1 & 78.1 & 78.1 \\
\hline K. pneumonia & 625 & 625 & 625 & 625 & 312.5 & 312.5 \\
\hline S. typhimurium & 625 & 625 & 156.2 & 156.2 & 156.2 & 156.2 \\
\hline P. aeruginosa & 5000 & 40000 & 78.1 & 156.2 & 78.1 & 156.2 \\
\hline
\end{tabular}


G3-vancomycin dendrimers showed a partial synergism $(0.5<$ FICI $<1)$ effect on $E$. coli with an FICI of 0.52 , while G5-vancomycin dendrimers exhibited a near synergism effect on this microorganism with an FICI value of almost 0.5. Both generations had only an indifference/additive mechanism $(1 \leq \mathrm{FICI} \leq 4)$ on Gram-positive bacteria $(\mathrm{FICI}=1)$. To sum up, synergic mechanism was only seen in Gram-negative bacteria in both generations.

\section{Results of stability studies}

Vancomycin UV spectra were defined in aqueous medium and in PBS (pH:7.4). As it is shown in Figure 2, UV-spectra of vancomycin hydrochloride in both media are similar and $\lambda_{\max }$ is appeared at 280 $\mathrm{nm}$ for both media.

Figure 3 shows a chromatogram of vancomycin hydrochloride solution which is assayed after 1 month storage at room temperature. As is shown, the retention time and height of the peak has not changed. It can be concluded that vancomycin is stable in this condition. Furthermore, our HPLC studies indicated that, vancomycin is a relatively stable molecule in harsh conditions (34).

Vancomycin-dendrimer complexes, injected after 1-month storage of the solution at room temperature, showed no significant change in AUC and retention time, compared to freshly prepared one and no new peak was appeared. The mean vancomycin concentration at time zero was $99.5 \pm$ 0.6 and $99.3 \pm 0.9 \mu \mathrm{g} / \mathrm{mL}$ for vancomycin G3 and vancomycin G5 respectively. These values changed to $99.0 \pm 0.7$ and $99.1 \pm 0.8 \mu \mathrm{g} / \mathrm{mL}$ after one month storage at ambient temperature $\left(25.0 \pm 1.0{ }^{\circ} \mathrm{C}\right)$. The statistical measurement done by one-way ANOVA showed no significant difference $(\mathrm{P}>0.05)$ in the amount of vancomycin between different formulations after 1 month stability studies.

Table 4. FICI of vancomycin with either G3 or G5 dendrimer combinations*

\begin{tabular}{|c|c|c|c|c|c|c|c|c|}
\hline \multirow[b]{2}{*}{ Bacteria } & \multicolumn{4}{|c|}{ Vancomycin/G3 systems } & \multicolumn{4}{|c|}{ Vancomycin/G5 systems } \\
\hline & $\begin{array}{l}\text { Vanc. } \\
\text { FIC }\end{array}$ & G3 FIC & FICI & Interaction & $\begin{array}{l}\text { Vanc. } \\
\text { FIC }\end{array}$ & G5 FIC & FICI $^{\text {a }}$ & Interaction \\
\hline S. aureus & 1 & 0.002 & 1.002 & Add $^{b}$ & 1 & 0.001 & 1.001 & Add \\
\hline MRSA & 1 & 0.002 & 1.002 & Add & 1 & 0.002 & 1.002 & Add \\
\hline E.coli & 0.5 & 0.02 & 0.52 & P.syn ${ }^{c}$ & 0.5 & 0.004 & 0.504 & Syn \\
\hline K. pneumonia & 1 & 0.03 & 1.03 & Add & 0.5 & 0.02 & 0.52 & P.syn \\
\hline S. typhimurium & 0.25 & 0.008 & 0.258 & Syn $^{d}$ & 0.25 & 0.008 & 0.258 & Syn \\
\hline P. aeruginosa & 0.02 & 0.004 & 0.024 & Syn & 0.02 & 0.004 & 0.024 & Syn \\
\hline
\end{tabular}

*: All tests were ran in triplicates and same FIC (or FICI) values were observed in the replications.

a) FICI: Fractional inhibitory concentration index

b) Add: additive (FICI $\geq 1)$

c) P.syn: Partial synergism $(0.5<\mathrm{FICI}<1)$

d) Syn: Synergism (FICI $\leq 0.5)$
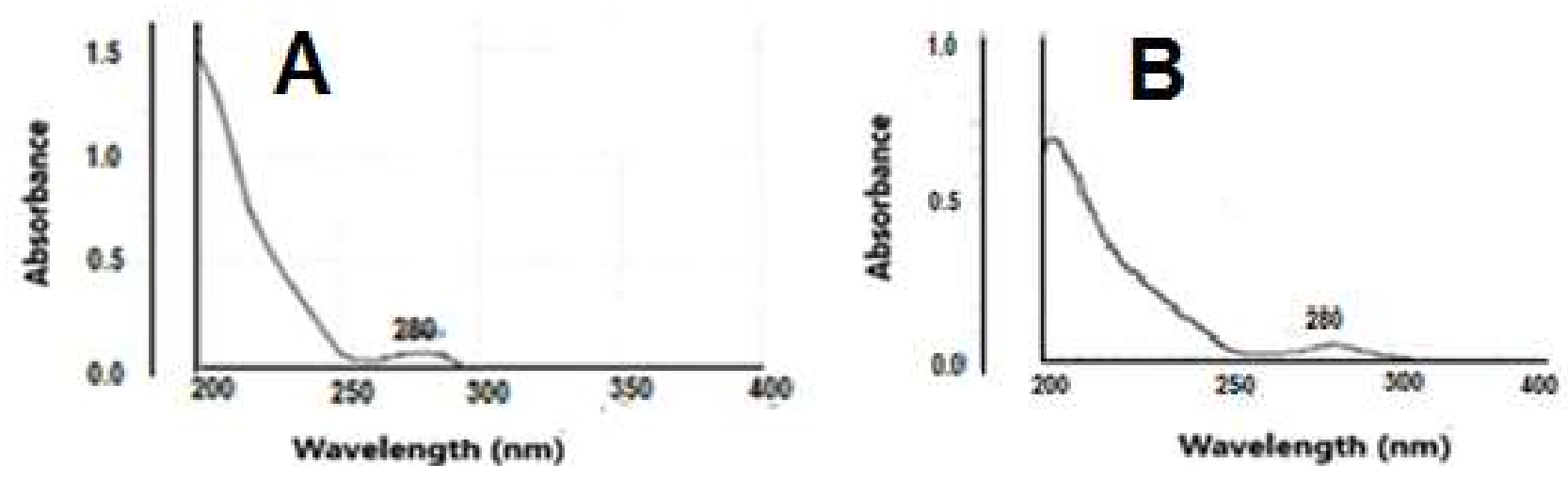

Figure 2. UV-spectrum of vancomycin hydrochloride in A) water and in B) PBS (pH: 7.4). 


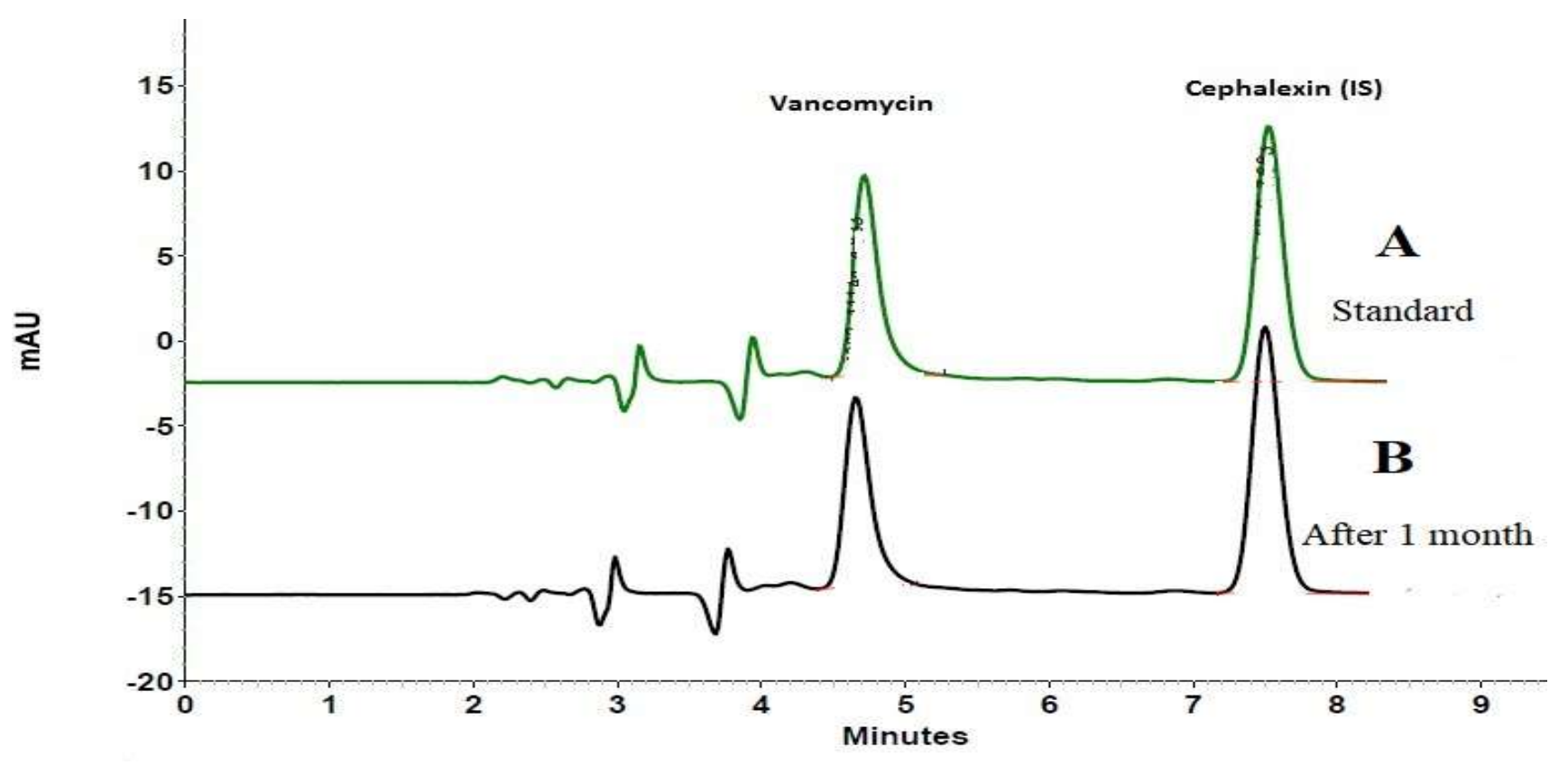

Figure 3. Vancomycin hydrochloride chromatograms A) freshly prepare, B) after 1 month storage at room temperature

ANOVA was followed by Tukey's HSD post hoc test and no significant difference $(\mathrm{P}>0.05)$ was observed between individual means.

\section{DISCUSSION}

Our results showed that the most sensitive microorganism toward vancomycin, with minimum MIC value, was $S$. aureus with an MIC of $2.4 \mu \mathrm{g} / \mathrm{mL}$. The MIC values for Gram-negative bacteria were more than $156.2 \mu \mathrm{g} / \mathrm{mL}$. The highest MIC value was for $P$. aeruginosa with an MIC and MBC values of 5000 and $40000 \mu \mathrm{g} / \mathrm{mL}$, respectively. As was explained earlier, vancomycin cannot penetrate the outer membrane of Gram-negative bacteria, due to its high molecular weight of about $1400 \mathrm{D}$ and hydrophilicity $(11,42,43)$. Gram-positive bacteria however, lack this outer membrane layer and therefore, as is confirmed by our results, vancomycin is effective against these bacteria while, it cannot show enough antibacterial effect on Gram-negative ones. In other word, Gram-negative bacteria are not responsive (sensitive) to vancomycin, because their outer membrane does not let this antibiotic enter the cell and reach its site of action. It seems reasonable that by weakening the barrier properties of this outer membrane layer of Gram-negative bacteria, one might overcome this limitation and make vancomycin an effective antibiotic against this kind of bacteria.

We used PAMAM dendrimer-vancomycin systems in order to broaden the spectrum of vancomycin activity. Our results revealed that dendrimer-vancomycin complex reduces MIC and MBC values up to 64 times (Table 3). The enhancement effects of dendrimers toward vancomycin depend on bacterial type and dendrimer generation, as is shown. Vancomycin dendrimers, in both cases (G3 and G5), significantly potentiated the drug effect on almost all Gram-negative bacteria. They reduced vancomycin MICs bye about 2, 2, 4 and 64 times for E. coli, $K$. pneumonia, $S$. typhimurium and $P$. aeruginosa, respectively. These improvements might be due to bacterial membrane perturbation or higher uptake of dendrimervancomycin systems, as discussed below.

Antibacterial effects of dendrimers have been reviewed by Boas and Heegaard (44) and discussed that cationic dendrimers show their antibacterial activity by adhering to anionic bacterial membrane and therefore, damaging it. Such damage may have been responsible for effects of PAMAM dendrimer on permeation of vancomycin observed here. It also might be due to increased local concentration of vancomycin. Such a mechanism has also been discussed by this group for covalently-bond 
dendrimer-drug for extracellular matrix-targeted local drug delivery (45).

Hong et al. (46), studied the effects of dendrimers on permeation of a model dye through an artificial membrane and $\mathrm{KB}$ rat cell lines and showed that dendrimers possibly reduce the barrier property of the membranes through hole formation or internalization of dendrimer-dye through endocytosis.

Another study showed G4 PAMAM dendrimer could permeabilize caco-2 cell monolayers (23). Guerin-Mechin et al. (47), observed changes in bacterial outer membrane fatty acid composition in response to treatment with quaternary ammonium compounds (QACs). Such mechanisms may have contributed to hole formation in bacterial outer membrane and subsequent increased delivery and entrance of vancomycin into its site of action.

In-vitro release of vancomycin from vancomycin dendrimers after $24 \mathrm{~h}$ showed that G5 and $\mathrm{G} 3$ vancomycin dendrimers released about $44 \%$ and $63 \%$ of their cargo into the receptor phase, while $100 \%$ of vancomycin was released from solution. The reduced vancomycin release from dendrimer systems could be due to a complex formation between dendrimers and the drug and hence, reduced vancomycin release from complexes. Our results are in good agreement with other groups $(30,48)$, who showed that dendrimers reduced release of ketoprofen and sulfamethoxazole, in comparison to the solutions, possibly due to complex formation between dendrimers and the drug. This might be because of high surface density of functional groups of dendrimers which can simultaneously interact with several drug molecules and entrapment of the drug in the internal void cavities. An inclusion box is made resulting from non-covalent interaction of vancomycin with either peripheral primary amino groups or interior voids of the dendrimer (44). As is explained by Cheng et al. (49) and Moghimi et al. (50), dendrimers can either physically entrap drugs
(Figure 4) or bind to drugs through chemical interactions (Figure 5).

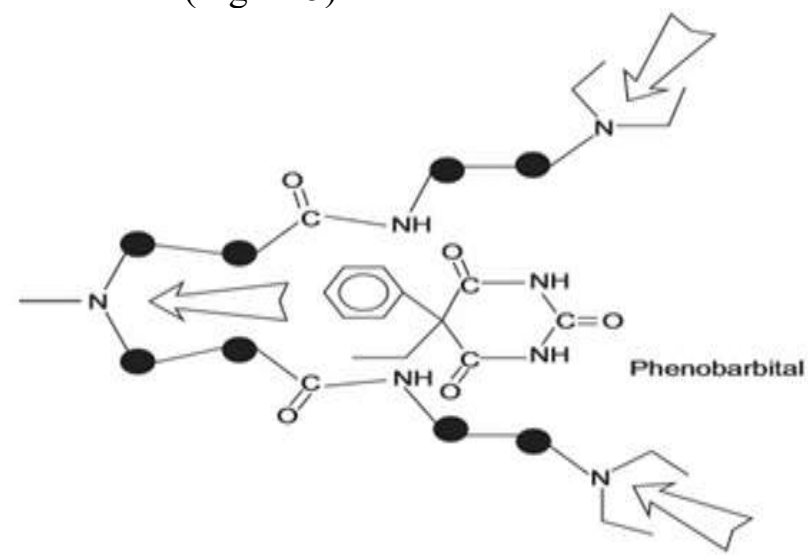

Figure 4. Localizations of phenobarbital molecules in the cavities of polyamidoamine (PAMAM) dendrimer; as described by Cheng et al. (49) (with permission from the corresponding author).

Our results also showed that the amounts of vancomycin released from G5 and G3 are different and that G5 showed lower release. This might be due to higher entrapment of vancomycin in G5 as a result of higher interacting sites in this dendrimer. Cheng and coworkers (49) demonstrated that higher generation PAMAM dendrimers can encapsulate more phenobarbital molecules (Figure 4), into the interior cavities, than lower generation dendrimer.

Our results showed that G5 vancomycindendrimer enhancement effect is either equal or higher than G3 based on MIC and FICI values (Table 3 and Table 4). Our results also revealed that G5 dendrimer showed synergistic effect with vancomycin against all Gram-negative bacteria (except for K. pneumonia with a partial synergistic effect), while the effect of G3 dendrimer was partial synergism and additive/indifference towards $E$. coli and $K$. pneumonia, respectively. G5 dendrimer showed lower FICI on E. coli and $K$. pneumonia of about 0.5 and 0.52 , respectively, compared to G3 dendrimers ( 0.52 and 1.03 correspondingly).
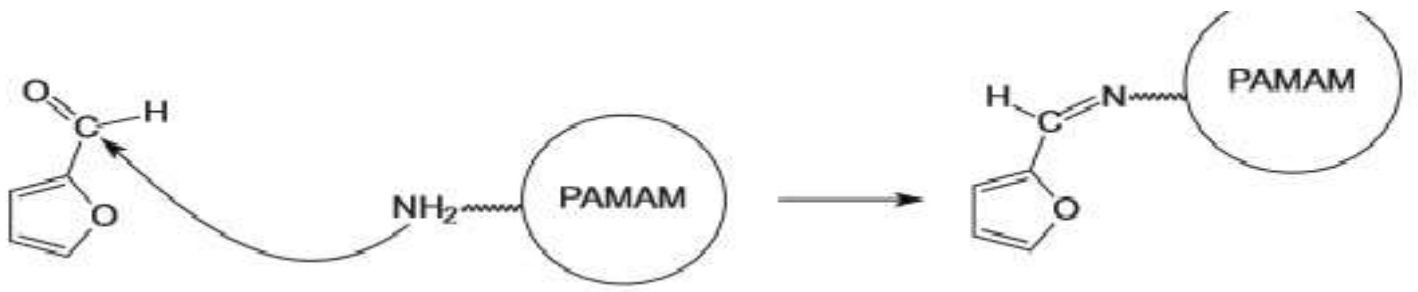

Figure 5. Probable chemical interaction between the surface nucleophilic (NH2) groups of polyamidoamine (PAMAM) dendrimer and furfural, proposed by Moghimi et al. (50). (with permission from the corresponding author). 
The difference between these two dendrimers may be due to difference in their interactions with vancomycin, (as was shown in release studies), difference in the interactions with the membrane or the size and entrapment efficiencies. However, more studies are needed to clarify the exact mechanism of this effect.

\section{CONCLUSION}

The present study revealed that both G3 and G5 cationic polyamidoamine (PAMAM) dendrimers are able to make Gram-negative bacteria sensitive to vancomycin, resulting in the decline of MIC values up to 64 times, possibly by increasing its permeation through bacterial outer membrane.

These findings are very interesting and promising in terms of broadening the spectrum of vancomycin activity. Such a strategy can be investigated and employed for other antibiotics and also can be used for increasing the life of these precious drugs.

As described in Introduction, there is a concern about toxicity of dendrimers. Fortunately, the concentration of PAMAM dendrimers employed here are low enough to decrease the risk of toxicity (19). Beside this, as explained earlier, it is possible to decrease toxicity of dendrimers by entrapping them in lipid nano carriers $(27,28)$, while the efficacy of dendrimer cargo is kept.

Further studies are in progress in our laboratories to find other methods for improving bacterial sensitivity (overcoming resistance) to antibiotics and broadening the spectrum of activity of existing antibiotics.

\section{ACKNOWLEDGMENT}

This work is a part of the $\mathrm{PhD}$ thesis of A. Serri at Shahid Beheshti University of Medical Sciences (SBMU). The Authors would like to thank Pharmaceutical Sciences Research Centre (SBMU), for their financial support.

\section{REFERENCES}

1. Cdc.gov. (2017). Antibiotic / Antimicrobial Resistance | CDC. [online] Available at: https://www.cdc.gov/drugresistance/index.html [Accessed 18 Jun. 2017].

2. Spellberg B, Powers JH, Brass EP, Miller LG, Edwards JE Jr. Trends in antimicrobial drug development: Implications for the future. Clin Infect Dis 2004; 38:1279-86.

3. Ventola CL. (2015). The antibiotic resistance crisis: Causes and threats. Pharmacy and Therapeutics, 40(4), 277-83.

4. DiMasi, J., Grabowski, H. and Hansen, R. (2016). Innovation in the pharmaceutical industry: New estimates of R\&D costs. Journal of Health Economics, 47, 20-33.

5. Tomasz, A. (1994). Multiple-Antibiotic-Resistant Pathogenic Bacteria -- A Report on the Rockefeller University Workshop. New England Journal of Medicine, 330(17), 1247-51.

6. Sheldrick, G., Jones, P., Kennard, O., Williams, D. and Smith, G. (1978). Structure of vancomycin and its complex with acetyl-D-alanyl-D-alanine. Nature, 271(5642),223-225.

7. Reynolds, P. (1989). Structure, biochemistry and mechanism of action of glycopeptide antibiotics. European Journal of Clinical Microbiology \& Infectious Diseases, 8(11),43-950.

8. Williams, D. (1996). The glycopeptide story - how to kill the deadly 'superbugs'. Nat. Prod. Rep., 13(6), 469-77.

9. Silhavy, T., Kahne, D. and Walker, S. (2010). The Bacterial Cell Envelope. Cold Spring Harbor Perspectives in Biology, 2(5), pp.a000414-a000414.

10. Delcour, A. (2009). Outer membrane permeability and antibiotic resistance. Biochimica et Biophysica Acta (BBA) - Proteins and Proteomics, 1794(5), 80816.

11. Zgurskaya, H., López, C. and Gnanakaran, S. (2015). Permeability Barrier of Gram-Negative Cell Envelopes and Approaches To Bypass It. ACS Infectious Diseases, 1(11), 512-22.

12. Watanakunakorn, C. (1984). Mode of action and invitro activity of vancomycin. Journal of Antimicrobial Chemotherapy, 14(suppl D), 7-18.

13. De Jong, (2008). Drug delivery and nanoparticles: Applications and hazards. International Journal of Nanomedicine, 133.

14. Zhang, L., Pornpattananangkul, D., Hu, C. and Huang, C. (2010). Development of Nanoparticles for Antimicrobial Drug Delivery. Current Medicinal Chemistry, 17(6), 585-94.

15. Chaniotakis, N., Thermos, K. and Kalomiraki, M. (2015). Dendrimers as tunable vectors of drug delivery systems and biomedical and ocular applications. International Journal of Nanomedicine, 1.

16. Sharma, A. and Kakkar, A. (2015). Designing Dendrimer and Miktoarm Polymer Based MultiTasking Nanocarriers for Efficient Medical Therapy. Molecules, 20(9) ,16987-17015.

17. Singh, J., Jain, K., Mehra, N. and Jain, N. (2016). Dendrimers in anticancer drug delivery: mechanism 
of interaction of drug and dendrimers. Artificial Cells, Nanomedicine, and Biotechnology, 44(7),1626-34.

18. Kesharwani, P., Banerjee, S., Gupta, U., Mohd Amin, M., Padhye, S., Sarkar, F. and Iyer, A. (2015). PAMAM dendrimers as promising nanocarriers for RNAi therapeutics. Materials Today, 18(10), 565-72.

19. Sadekar, S. and Ghandehari, H. (2012). Transepithelial transport and toxicity of PAMAM dendrimers: Implications for oral drug delivery. Advanced Drug Delivery Reviews, 64(6), 571-88.

20. Kumar, A., Yellepeddi, V., Davies, G., Strychar, K. and Palakurthi, S. (2010). Enhanced gene transfection efficiency by polyamidoamine (PAMAM) dendrimers modified with ornithine residues. International Journal of Pharmaceutics, 392(1-2), 294-303.

21. Rao, N., Sunkara, M., Amreddy, N., Kurra, V. and Adimoolam, M. (2015). Photosensitizer and peptideconjugated PAMAM dendrimer for targeted in vivo photodynamic therapy. International Journal of Nanomedicine, 6865.

22. Bahadır, E. and Sezgintürk, M. (2016). Poly(amidoamine) (PAMAM): An emerging material for electrochemical bio(sensing) applications. Talanta, 148, 427-38.

23. El-Sayed, M., Ginski, M., Rhodes, C. and Ghandehari, H. (2002). Transepithelial transport of poly(amidoamine) dendrimers across Caco-2 cell monolayers. Journal of Controlled Release, 81(3), 355-65.

24. Thiagarajan, G., Greish, K. and Ghandehari, H. (2013). Charge affects the oral toxicity of poly(amidoamine) dendrimers. European Journal of Pharmaceutics and Biopharmaceutics, 84(2),330-34.

25. Kitchens, K., Foraker, A., Kolhatkar, R., Swaan, P. and Ghandehari, H. (2007). Endocytosis and Interaction of Poly (Amidoamine) Dendrimers with Caco-2 Cells. Pharmaceutical Research, 24(11),2138-45.

26. Yellepeddi, V. and Ghandehari, H. (2016). Poly(amido amine) dendrimers in oral delivery. Tissue Barriers, 4(2), e1173773.

27. Movassaghian, S., Moghimi, H., Shirazi, F. and Torchilin, V. (2011). Dendrosome-dendriplex inside liposomes: as a gene delivery system. Journal of Drug Targeting, 19(10), 925-32.

28. Movassaghian, S., Moghimi, H., Shirazi, F., koshkaryev, A. and Torchilin, V. (2013). Efficient down-regulation of PKC-gene expression in A549 lung cancer cells mediated by antisense oligodeoxynucleotides in dendrosomes. International Journal of Pharmaceutics, 441,82-91.

29. Cheng, Y., Qu, H., Ma, M., Xu, Z., Xu, P., Fang, Y. and $\mathrm{Xu}, \mathrm{T}$. (2007). Polyamidoamine (PAMAM) dendrimers as biocompatible carriers of quinolone antimicrobials: An in vitro study. European Journal of Medicinal Chemistry, 42(7), 1032-38.
30. Ma, M., Cheng, Y., Xu, Z., Xu, P., Qu, H., Fang, Y., $\mathrm{Xu}, \mathrm{T}$. and Wen, L. (2007). Evaluation of polyamidoamine (PAMAM) dendrimers as drug carriers of anti-bacterial drugs using sulfamethoxazole (SMZ) as a model drug. European Journal of Medicinal Chemistry, 42(1), 93-8.

31. Winnicka, K., Wroblewska, M., Wieczorek, P., Sacha, P. and Tryniszewska, E. (2013). The Effect of PAMAM Dendrimers on the Antibacterial Activity of Antibiotics with Different Water Solubility. Molecules, 18(7), ,8607-17.

32. Choi, S., Myc, A., Silpe, J., Sumit, M., Wong, P., McCarthy, K., Desai, A., Thomas, T., Kotlyar, A., Holl, M., Orr, B. and Baker, J. (2013). DendrimerBased Multivalent Vancomycin Nanoplatform for Targeting the Drug-Resistant Bacterial Surface. ACS Nano, 7(1),214-28.

33. Sonawane, S., Kalhapure, R., Rambharose, S., Mocktar, C., Vepuri, S., Soliman, M. and Govender, T. (2016). Ultra-small lipid-dendrimer hybrid nanoparticles as a promising strategy for antibiotic delivery: In vitro and in silico studies. International Journal of Pharmaceutics, 504(1-2), 1-10.

34. Serri, a., Moghimi, H., Mahboubi, A. and Zarghi, A. (2017). Stability-indicating HPLC method for determination of vancomycin hydrochloride in pharmaceutical dosage forms. Acta Poloniae Pharmaceutica, 74(1), 73-9.

35. Sigma-Aldrich. (2017). PAMAM Dendrimers Dendrimers | Sigma-Aldrich. [online] Available at: http://www.sigmaaldrich.com/materialsscience/material-science-

products.html? TablePage $=9539880 \quad$ [Accessed 30 Apr. 2017].

36. Hariri, E., Mahboubi, A. and Kobarfard, F. (2016). Synthesis and antibacterial activity of novel hydroxy semicarbazone derivatives. Iranian Journal of Pharmaceutical reserech, [online] 15(Suppl),.29-35.

37. CLSI. Methods for Dilution Antimicrobial Susceptibility Tests $\mathrm{f}$ or Bacteria That Grow Aerobically; Approved Standard-Ninth Edition. CLSI document M07-A9. Wayne, PA: Clinical and Laboratory Standards Institute; 2012.

38. CLSI. Performance Standards for Antimicrobial Susceptibility Testing; Twenty-Fifth Informational Supplement. CLSI document M100-S25. Wayne, PA: Clinical and Laboratory Standards Institute; 2015.

39. Mahboubi (2012). Evaluation of antibacterial activity of three Iranian medicinal plants. African Journal of Microbiology Research, 6(9).

40. Mahboubi, A., Asgarpanah, J., Sadaghiyani, P. and Faizi, M. (2015). Total phenolic and flavonoid content and antibacterial activity of Punica granatum L. var. pleniflora flowers (Golnar) against bacterial strains causing foodborne diseases. $B M C$ Complementary and Alternative Medicine, 15(1). 
41. Bonapace, C., Bosso, J., Friedrich, L. and White, R. (2002). Comparison of methods of interpretation of checkerboard synergy testing. Diagnostic Microbiology and Infectious Disease, 44(4), 363-66.

42. Hammes, W. and Neuhaus, F. (1974). On the Mechanism of Action of Vancomycin: Inhibition of Peptidoglycan Synthesis in Gaffkya homari. Antimicrobial Agents and Chemotherapy, 6(6), 72228.

43. Novikova, O. and Solovyeva, T. (2009). Nonspecific porins of the outer membrane of Gram-negative bacteria: Structure and functions. Biochemistry (Moscow) Supplement Series A: Membrane and Cell Biology, 3(1), 3-15.

44. Boas, U. and Heegaard, P. (2004). Dendrimers in Drug Research. ChemInform, 35(16).

45. Sakharov, D., Jie, A., Bekkers, M., Emeis, J. and Rijken, D. (2001). Polylysine as a Vehicle for Extracellular Matrix-Targeted Local Drug Delivery, Providing High Accumulation and Long-Term Retention Within the Vascular Wall. Arteriosclerosis, Thrombosis, and Vascular Biology, 21(6), pp.943-48.

46. Hong, S., Bielinska, A., Mecke, A., Keszler, B., Beals, J., Shi, X., Balogh, L., Orr, B., Baker, J. and Banaszak Holl, M. (2004). Interaction of Poly(amidoamine) Dendrimers with Supported Lipid Bilayers and Cells: Hole Formation and the Relation to Transport. Bioconjugate Chemistry, 15(4), 774-82.

47. Guerin-Mechin, L., Dubois-Brissonnet, F., Heyd, B. and Leveau, J. (2000). Quaternary ammonium compound stresses induce specific variations in fatty acid composition of Pseudomonas aeruginosa. International Journal of Food Microbiology, 55(1-3), 157-59.

48. Na, M., Yiyun, C., Tongwen, X., Yang, D., Xiaomin, W., Zhenwei, L., Zhichao, C., Guanyi, H., Yunyu, S. and Longping, W. (2006). Dendrimers as potential drug carriers. Part II. Prolonged delivery of ketoprofen by in vitro and in vivo studies. European Journal of Medicinal Chemistry, 41(5). 670-74.

49. Cheng, Y., Li, Y., Wu, Q., Zhang, J. and $\mathrm{Xu}, \mathrm{T}$. (2009). encapsulation/electrostatic phenobarbital molecules dendrimers: Evidence from 2D-NOESY investigations. European Journal of Medicinal Chemistry, 44(5), 2219-23.

50. Moghimi, H., Varshochian, R., Kobarfard, F. and Erfan, M. (2009). Reduction of percutaneous absorption of toxic chemicals by dendrimers. Cutaneous and Ocular Toxicology, 29(1), 34-40. 